\title{
Smoking Behavior of Women Before, During, and after Pregnancy: Indicators of Smoking, Quitting, and Relapse
}

\author{
Tessa Scheffers-van Schayck ${ }^{a}$ b Marlous Tuithofa ${ }^{a}$ Roy Otten ${ }^{c-e}$ Rutger Engels ${ }^{f}$ \\ Marloes Kleinjan ${ }^{a, g}$ \\ ${ }^{a}$ Trimbos Institute, Netherlands Institute of Mental Health and Addiction, Epidemiology and Research Support, \\ Utrecht, The Netherlands; ${ }^{b}$ Department of Developmental Psychology, Utrecht University, Utrecht, The \\ Netherlands; ' ${ }^{C}$ Research and Development, Pluryn, Nijmegen, The Netherlands; ${ }^{\mathrm{d}}$ ASU REACH Institute, Department \\ of Psychology, Arizona State University, Tempe, AZ, USA; e Developmental Psychopathology, Radboud University, \\ Nijmegen, The Netherlands; ${ }^{\mathrm{f}}$ Executive Board, Erasmus University, Rotterdam, The Netherlands; ${ }^{9}$ Department of \\ Interdisciplinary Social Sciences, Utrecht University, Utrecht, The Netherlands
}

\section{Keywords \\ Smoking · Pregnancy $\cdot$ Cessation $\cdot$ Relapse $\cdot$ Monitor}

\section{Abstract}

Background: Smoking cessation during pregnancy and preventing relapse postpartum is a pivotal public health priority. Objectives: This study examined the risk and protective indicators of women who (a) smoke before pregnancy, (b) smoke during the entire pregnancy, (c) successfully quit smoking during pregnancy, and (d) relapse postpartum. Method: This paper reports secondary analyses of the Dutch population-based Monitor on Substance Use and Pregnancy (2016). A representative sample of mothers of young children $(n=1,858)$ completed questionnaires at youth health care centers. Bivariate and multivariate logistic regression analyses were conducted. Results: Main results showed that women's smoking around pregnancy was strongly associated with the partner's smoking status before pregnancy, partner's change in smoking during pregnancy, and partner's change in smoking postpartum. Women's educational level and cannabis use before pregnancy were also related with women's smoking before and during pregnancy. Women's intensity of alcohol use before pregnancy was ambiguously related with women's smoking before and during pregnancy. Conclusions: One of the key findings of this study suggests that it is essential that partners quit smoking before pregnancy and do not smoke during pregnancy. If partners continue smoking during pregnancy, they should quit smoking postpartum. Health care professionals can play an important role in addressing partners' smoking and giving them evidence-based cessation support before, during, and after pregnancy.

\footnotetext{
(c) 2019 The Author(s) Published by S. Karger AG, Basel
}

\section{Introduction}

Smoking during pregnancy and relapse postpartum is a major public health concern $[1,2]$. Smoking during pregnancy has detrimental health effects on women's pregnancies (e.g., pre-term birth and low birth weight) [3] as well as on infants' and children's physical health and cognitive and social development (e.g., neurodevel-

Tessa Scheffers-van Schayck

Trimbos Institute, Netherlands Institute of Mental Health and Addiction Epidemiology and Research Support

Da Costakade 45, Utrecht, 3521 VS (The Netherlands)

E-Mail tscheffers@trimbos.nl 
opmental and behavioral problems) [3-6]. In addition, children's exposure to secondhand smoke has been associated with, among other risks, increased frequency of asthma $[3,7]$ and an increased risk that children will start smoking in the future [8].

Pregnancy could serve as a teachable moment for women to quit smoking [9]. This argument is supported by recent research showing that women are more likely to quit smoking during pregnancy than before pregnancy [10]. Nonetheless, many women continue to smoke during pregnancy. A large study using representative national samples from 54 low- and middle-income countries provided evidence that the pooled prevalence of any tobacco use in pregnancy was $2.6 \%$, with the highest prevalence in Turkey (15.0\%) [11]. Moreover, postpartum relapse rates of women who quit smoking during pregnancy are also high. A meta-analysis showed that $43 \%$ of women who stopped smoking during pregnancy began smoking again at 6 months postpartum [12]. In addition, subgroup analyses of this meta-analysis revealed that the rate of women who relapsed at 6 months postpartum increased to $74 \%$ when only studies with biochemically validated abstinence data were included [12].

Multiple smoking cessation and relapse prevention interventions for pregnant women have been developed and tested [13-15], but evidence for their effectiveness is mixed. A Cochrane review illustrated that psychosocial interventions could decrease the number of women who smoke in late pregnancy by $35 \%$ [16]. In addition, financial incentives and interventions that included feedback (e.g., information about fetal health status) have proven effective in reducing the prevalence of smoking women in late pregnancy [16]. In contrast, postpartum relapse prevention interventions have shown limited effects $[2,17]$. It seems that many women and their offspring do not receive the maximum health benefits of smoking cessation [12]. Therefore, there is still considerable room for improvement with regard to cessation and relapse-prevention interventions in pregnancy. Effectiveness might be improved by tailoring interventions toward specific characteristics, risk and protective indicators, and needs of the target group. Multiple systematic reviews and meta-analyses have shown that tailored (vs. non-tailored) health promotion interventions are more effective [18-20].

In order to provide women who smoke with tailored and (cost-)effective help to quit smoking before and during pregnancy and to avoid postpartum relapse, a profound understanding of this specific population is essential. So far, many studies have examined different charac-

Indicators of Women's Smoking Behavior before, During, and after Pregnancy teristics of women who either (1) smoked before or during pregnancy, (2) quit smoking during pregnancy, or (3) relapsed postpartum. A large study focusing on smoking before pregnancy found that women who did not take folic acid, who had a low educational level, who had an unplanned pregnancy, and who lived alone were more likely to smoke before pregnancy [21]. Other studies found that women who were younger [22-24], were unmarried, or lived alone $[21,23,25]$, had a low educational level [2126], lived in an extended family [23], did not take folic acid [21], and had partners who smoked [25] were more likely to smoke during pregnancy. In addition, women were more likely to quit smoking during pregnancy when they were married [27], had a higher socioeconomic status [22], had a higher educational level [27-29], lived with a non-smoking cohabitant $[27,29]$, and smoked fewer cigarettes before pregnancy or quitting [27]. Finally, women were more likely to relapse postpartum when they quit smoking during instead of before pregnancy [30, 31], did not breastfeed [30,32], had a smoking environment at home [30, 32], had more family and friends who smoked, had a partner that continued smoking during pregnancy, smoked more cigarettes per day before pregnancy, and were younger and unmarried [31]. To conclude, the majority of studies solely examined the associations between risk indicators and women's smoking behavior with respect to one or 2 moments around women's pregnancy (e.g., before or during pregnancy). Only 2 studies examined risk and protective indicators at all points around pregnancy (i.e., before, during, and after) in the same study. However, the data in these 2 studies were collected more than 20 years ago and lacked findings on smoking during pregnancy [33] or combined smoking before and during pregnancy [34]. Moreover, most studies mainly focused on sociodemographic indicators and less on partner's smoking status or other maternal substance use (e.g., alcohol and cannabis) around pregnancy. The combination of looking at all three points around pregnancy and including various indicators provides a more complete picture of whether (change in) behavior of women and their context before pregnancy is associated with smoking of women later on during or after pregnancy.

The current study uses cross-sectional data of a representative population-based study from the Netherlands (i.e., the Monitor on Substance Use and Pregnancy) to exploratively examine demographic, substance-use-related, and pregnancy-related risk and protective indicators of women who (a) smoke before pregnancy, (b) smoke during the entire pregnancy, (c) successfully quit smoking during pregnancy, and (d) relapse postpartum. 
The use of a large representative sample, a broad spectrum of indicators (i.e., sociodemographic, partner's smoking behavior around pregnancy, and other maternal substance use), and the measurement of 3 time points around pregnancy helps to gain insight into the characteristics of women who smoke around pregnancy and provides information on whether the characteristics differ over the 3 time points around pregnancy. These insights may contribute to the development of tailored cessation and relapse-prevention interventions.

\section{Materials and Methods}

\section{Sample}

The Dutch Monitor on Substance Use and Pregnancy [35] is a representative population-based cross-sectional study in which mothers have to answer questions retrospectively about their substance use (i.e., smoking, drinking, and drug use) before, during, and after pregnancy. For the present study, we used only the data collected in 2016 (the Monitor will be repeated in 2018). In the Netherlands, all children up to 18 years old receive preventive health care at youth health care (YHC) centers [36]. Therefore, to collect a sample that properly represented mothers with young children (i.e., youngest child younger than 5 years) and to maximize the chance that mothers with a low socioeconomic status would participate in this study, mothers were recruited at YHC centers. A total of 34 cities across the Netherlands were randomly selected to be approached for participation in the Monitor. In total, $27(79.4 \%)$ of these cities agreed to participate, and their YHC centers were included. For the 7 cities that did not want to participate, other cities of similar urbanicity in the same region were approached, and all YHC centers in these cities registered for participation. In total, 46 YHC centers, distributed throughout 35 cities in the Netherlands, participated.

The cluster sampling strategy resulted in a representative sample of Dutch mothers with young children with some small deviations. For instance, the percentage of Western mothers in this sample was higher than that of the entire Dutch population (86.8 vs. $80.5 \%)$. After being weighted for age, educational level, and ethnicity, the sample properly represented Dutch mothers with young children. In total, 1,858 mothers (M 31.69, SD 4.69) were included. The average age of the youngest child was 11.14 months (SD 12.22).

\section{Data Collection}

Data were collected in November and December 2016. Mothers who visited a YHC center with their child were asked whether they would like to participate in a study on health behaviors of mothers of young children. To participate, participants needed to be a mother of children aged $0-4$ years. There were no exclusion criteria. Mothers received oral instructions by trained research assistants and gave permission to participate in our study (active informed consent). Participation included that mothers filled out a short questionnaire ( $\sim \mathrm{min})$ at the YHC center while waiting for their appointment. The response rate was $94.2 \%$. Some mothers declined to participate because they could not speak Dutch fluently. Mothers who did not agree to participate due to a lack of time were offered the opportunity to fill out a paper or online version of the questionnaire later at home. Participants filled out the questionnaire on a tablet $(84.4 \%)$, paper $(13.6 \%)$, or online $(2.0 \%)$. Because of the sensitivity of this study's topic and to minimize socially desirable answers, research assistants approached the mothers and emphasized that the data would be collected and processed anonymously.

\section{Measures}

In the questionnaire, maternal smoking status was assessed with respect to three different time points around the pregnancy: before, during, and after pregnancy. With respect to the maternal status before pregnancy, mothers were asked whether they had smoked in the four weeks before their pregnancy. Smoking during pregnancy was measured for each trimester separately (1-3 months; 4-6 months; 7-9 months), by asking mothers whether they had smoked at all during each trimester. Smoking status after pregnancy was assessed by asking whether they had smoked in the past four weeks. Four groups were constructed (Fig. 1). From the total sample, the first group consisted out of women who smoked in the four weeks before pregnancy (reference group: women who did not smoke before pregnancy). From this first group, a second group was selected including women who successfully quit smoking at some point during pregnancy through the end of the pregnancy (i.e., did not smoke in the third trimester; reference group: women who did not successfully quit smoking during pregnancy). From the second group we selected a third group, consisting out of women who had successfully quit smoking during pregnancy, but relapsed postpartum (reference group: women who successfully quit smoking during pregnancy and remained smoke-free postpartum). Finally, a fourth group was constructed, including women who smoked during the entire pregnancy (i.e., all three trimesters; reference group: women who did not smoke or who only smoked before pregnancy or who smoked less than three trimesters during pregnancy). Women could belong to more than one group. For instance, women who successfully quit smoking at some point during the pregnancy could also belong to the group of women who relapsed after pregnancy (Fig. 1)."

Several maternal sociodemographic indicators were assessed, including age (four categories), educational level (low: primary education or pre-secondary vocational education/medium: secondary (vocational) education/high: higher vocational education or university), ethnicity (Dutch/other-Western/non-Western), and living situation (partner and children/other). The following risk and protective indicators were measured: 1) breastfeeding (yes/ no); 2) intake of folic acid before and during pregnancy (yes/no); 3) intensity of alcohol use before pregnancy (i.e., number of glasses alcohol consumed on days that women drank alcohol before pregnancy: non-drinkers, 1 glass, $\geq 2$ glasses); 4) alcohol use during pregnancy (yes/no); 5) cannabis use before pregnancy (yes/no); 6) partner's smoking status before pregnancy (yes/no); 7) change in partner's smoking behavior during pregnancy (compared to before pregnancy: partner did not smoke before and during pregnancy/ partner's smoking decreased or partner quit smoking during pregnancy/partner smoked as much or more during pregnancy); 8) change in partner's smoking behavior postpartum (compared to before and during pregnancy: partner did not smoke before, during, and after pregnancy/partner smoked before or during pregnancy but not after pregnancy/partner smoked after pregnancy); 9) talked about smoking cessation with a health care professional 


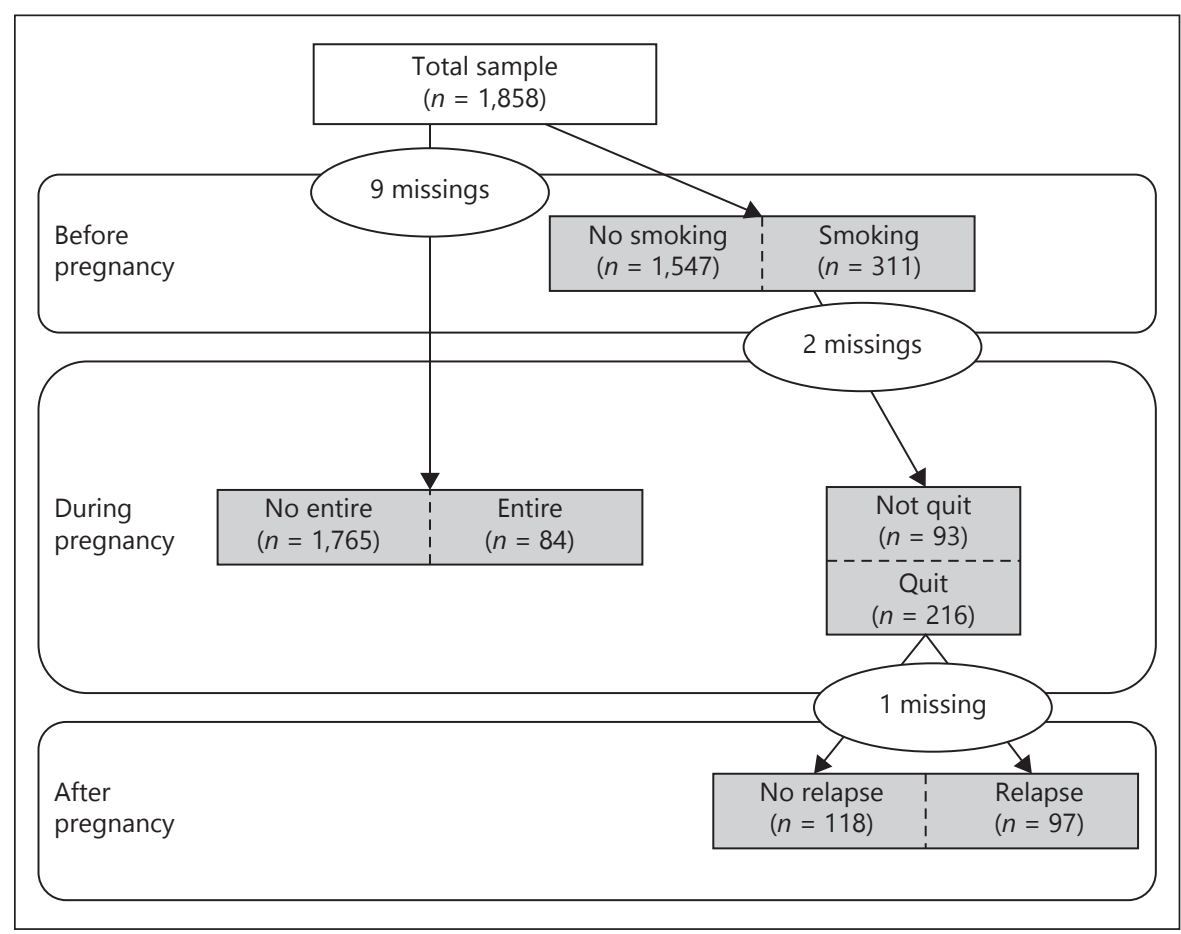

Fig. 1. The 4 groups of women that were constructed to include in the analyses.

during pregnancy (yes/no); and 10) use of smoking cessation support during pregnancy (yes/no). Breastfeeding included any breastfeeding without a required minimum duration. Use of smoking cessation support during pregnancy (e.g., nicotine replacement therapy, behavioral coaching, and e-health interventions) was categorized "yes" if mothers checked at least one of the cessation support answer options on the question whether they had used any of the following smoking cessation support options. If mothers indicated that they had not used any support, it was categorized "no". Because this study aimed to be nationally representative and the data were collected when mothers were waiting for their appointment at the YHC centers, the questionnaire was very short and no data were collected on e.g. nicotine dependence, mental health disorders or other indicators related to smoking behavior.

\section{Statistical Analyses}

In order to control for the clustered sampling procedure and to ensure that the data were representative of Dutch women with young children, all analyses were carried out using the Complex Samples Module of the software program Statistical Package for the Social Sciences, version 20. Because most questionnaires were collected digitally, missing data was not an issue. First, frequencies were calculated to provide characteristics of the 4 groups. Note that these groups could not be compared, as women could belong to 2 or more groups. Next, to determine the most important indicators related to the 4 groups, a 2-stage approach was carried out. First, bivariate logistic regression models were used for each outcome variable. If the $p$ value of the bivariate model (BM) was lower than 0.05 , it was considered statistically significant. Second, multivariate logistic regression models were built for the 4 groups. The significant indicators from the BM were entered simultaneously in the multivariate models (MM). Adjusted ORs, 95\% CIs, and values are reported.

Indicators of Women's Smoking Behavior before, During, and after Pregnancy

\section{Results}

Table 1 presents the weighted descriptive statistics for the risk and protective indicators for the total sample and for each group. Of the 1,858 women who participated in this study, 358 women (20.2\%) reported that they had smoked at some time around pregnancy. More specifically, $17.4 \%$ smoked before pregnancy, $4.9 \%$ smoked during the entire pregnancy, $12.0 \%$ successfully quit smoking during pregnancy, and 5.5\% successfully quit smoking during pregnancy but relapsed postpartum. Of the women who successfully quit smoking during pregnancy, $46.1 \%$ relapsed postpartum. In total, $82.3 \%$ of these women relapsed within 6 months postpartum.

\section{Indicators Associated with Women who Smoked before Pregnancy}

Table 2 presents the $\mathrm{BM}$ and $\mathrm{MM}$ of risk and protective indicators that were associated with women who smoked before pregnancy. According to the BM, women who smoked before pregnancy were younger, less educated, and lived without a partner more often compared to women who did not smoke before pregnancy. In addition, women who smoked before pregnancy less often took folic acid before pregnancy, more often used substances before pregnancy (i.e., higher intensity of alcohol use, any cannabis use), and more often had a partner who 
Table 1. Descriptive statistics for demographic and other indicators for total sample and each group

\begin{tabular}{|c|c|c|c|c|c|}
\hline Indicators & $\begin{array}{l}\text { Total } \\
\text { sample, } \\
n(\%)\end{array}$ & $\begin{array}{l}\text { Women who } \\
\text { smoked BP, } \\
n(\%)^{*}\end{array}$ & $\begin{array}{l}\text { Women who } \\
\text { smoked during } \\
\text { the entire } \\
\text { pregnancy, } n(\%)^{*}\end{array}$ & $\begin{array}{l}\text { Women who } \\
\text { successfully quit } \\
\text { smoking DP, } n(\%)^{*}\end{array}$ & $\begin{array}{l}\text { Women who } \\
\text { relapsed PP, } n \\
(\%)^{*}\end{array}$ \\
\hline Number & 1,858 & 311 & 84 & 216 & 97 \\
\hline \multicolumn{6}{|l|}{ Maternal age, years } \\
\hline $18-24$ & $127(8.7)$ & $44(17.0)$ & $14(20.4)$ & $25(14.1)$ & $8(9.6)$ \\
\hline $30-34$ & $739(38.4)$ & $100(30.1)$ & $20(23.0)$ & $80(34.2)$ & $33(30.8)$ \\
\hline $35+$ & $528(24.4)$ & $65(17.1)$ & $22(21.8)$ & $42(15.7)$ & $18(14.8)$ \\
\hline \multicolumn{6}{|l|}{ Educational level } \\
\hline Low & $153(11.8)$ & $48(19.8)$ & $26(36.4)$ & $23(14.6)$ & $13(18.8)$ \\
\hline Medium & $705(38.1)$ & $170(52.5)$ & $50(55.1)$ & $110(49.3)$ & $57(53.7)$ \\
\hline High & $1,000(50.1)$ & $93(27.7)$ & $8(8.4)$ & $83(36.1)$ & $27(27.5)$ \\
\hline \multicolumn{6}{|l|}{ Living situation } \\
\hline Dutch & $1,469(69.4)$ & $256(74.7)$ & $71(79.0)$ & $175(72.4)$ & $78(71.6)$ \\
\hline Other Western & $144(10.6)$ & $26(11.4)$ & $6(9.6)$ & $19(12.1)$ & $6(9.0)$ \\
\hline Other non-Western & $244(20.0)$ & $29(13.9)$ & $7(11.4)$ & $22(15.5)$ & $13(19.4)$ \\
\hline \multicolumn{6}{|l|}{ Intake of folic acid BP } \\
\hline No & $569(33.1)$ & $130(44.5)$ & $24(32.6)$ & $99(48.3)$ & $39(44.8)$ \\
\hline Yes & $1,289(66.9)$ & $181(55.5)$ & $60(67.4)$ & $117(51.7)$ & $58(55.2)$ \\
\hline \multicolumn{6}{|l|}{ Intake of folic acid DP } \\
\hline No & $133(8.6)$ & $23(8.3)$ & $11(14.6)$ & $12(6.1)$ & $4(5.0)$ \\
\hline Yes & $1,718(91.4)$ & $286(91.7)$ & $73(85.4)$ & $204(93.9)$ & $93(95.0)$ \\
\hline \multicolumn{6}{|l|}{ Breastfeeding } \\
\hline No & $490(26.1)$ & $123(38.9)$ & $45(50.8)$ & $71(32.2)$ & $35(35.7)$ \\
\hline Yes & $1,351(73.9)$ & $185(61.1)$ & $39(49.2)$ & $144(67.8)$ & $62(64.3)$ \\
\hline \multicolumn{6}{|l|}{ Alcohol use DP } \\
\hline \multicolumn{6}{|l|}{ Cannabis use $\mathrm{BP}$} \\
\hline No & $1,818(97.8)$ & $285(90.2)$ & $78(91.3)$ & $200(90.7)$ & $87(86.3)$ \\
\hline Yes & $33(2.2)$ & $25(9.8)$ & $6(8.7)$ & $16(9.3)$ & $10(13.7)$ \\
\hline \multicolumn{6}{|l|}{ Smoking of partner BP } \\
\hline No & $1,345(71.1)$ & $97(30.8)$ & $18(23.4)$ & $76(34.1)$ & $29(27.8)$ \\
\hline Yes & $510(28.9)$ & $214(69.2)$ & $66(76.6)$ & $140(65.9)$ & $68(72.2)$ \\
\hline \multicolumn{6}{|l|}{$\begin{array}{l}\text { Change in partner's smoking DP } \\
\text { compared to BP }\end{array}$} \\
\hline Did not smoke BP and DP & $1,335(71.7)$ & $106(34.6)$ & $21(26.7)$ & $82(38.2)$ & $31(30.7)$ \\
\hline Decreased or quit & $230(13.0)$ & $92(29.4)$ & $22(24.7)$ & $67(31.3)$ & $29(32.1)$ \\
\hline As much as or increased & $268(15.3)$ & $107(36.0)$ & $41(48.6)$ & $62(30.5)$ & $35(37.2)$ \\
\hline \multicolumn{6}{|l|}{ Change in partner's smoking PP } \\
\hline Did not smoke at all & $1,336(72.3)$ & $124(41.2)$ & $28(36.5)$ & $95(44.6)$ & $39(39.4)$ \\
\hline Smoked BP or DP, but not PP & $76(4.1)$ & $24(7.0)$ & $4(4.5)$ & $21(8.9)$ & $2(1.8)$ \\
\hline Smoked PP & $419(23.6)$ & $159(51.8)$ & $52(59.0)$ & $98(46.5)$ & $55(58.8)$ \\
\hline \multicolumn{6}{|l|}{$\begin{array}{l}\text { Talked about smoking cessation } \\
\text { with health care professionals DP }\end{array}$} \\
\hline No & $253(80.3)$ & $249(80.9)$ & $48(56.9)$ & $197(92.3)$ & $84(91.0)$ \\
\hline Yes & $59(19.7)$ & $56(19.1)$ & $36(43.1)$ & $15(7.7)$ & $9(9.0)$ \\
\hline
\end{tabular}


Table 1. (continued)

\begin{tabular}{|c|c|c|c|c|c|}
\hline Indicators & $\begin{array}{l}\text { Total } \\
\text { sample, } \\
n(\%)\end{array}$ & $\begin{array}{l}\text { Women who } \\
\text { smoked BP, } \\
n(\%)^{*}\end{array}$ & $\begin{array}{l}\text { Women who } \\
\text { smoked during } \\
\text { the entire } \\
\text { pregnancy, } n(\%)^{*}\end{array}$ & $\begin{array}{l}\text { Women who } \\
\text { successfully quit } \\
\text { smoking DP, } n(\%)^{*}\end{array}$ & $\begin{array}{l}\text { Women who } \\
\text { relapsed PP, } n \\
(\%)^{*}\end{array}$ \\
\hline \multicolumn{6}{|c|}{ Use of smoking cessation support DP } \\
\hline No & $295(93.6)$ & $288(94.0)$ & $74(89.2)$ & $207(96.8)$ & $91(95.5)$ \\
\hline Yes & $22(6.4)$ & $20(6.0)$ & $10(10.8)$ & $8(3.2)$ & $5(4.5)$ \\
\hline
\end{tabular}

smoked before pregnancy. Most associations remained significant in the MM except younger age and the use of folic acid before pregnancy.

\section{Indicators Associated with Women who Smoked during the Entire Pregnancy}

The BM shows that women who smoked during the entire pregnancy were younger, less educated and lived without a partner more often than women who did not smoke during the entire pregnancy (Table 2). In addition, they were more likely to have a lower intensity of alcohol use before pregnancy and use any cannabis before pregnancy than women who did not smoke during the entire pregnancy. Lastly, women who did (vs. did not) smoke during the entire pregnancy were more likely to have a partner who smoked during the pregnancy. In the MM, low educational level, living without a partner, and smoking of the partner during pregnancy remained significantly associated with smoking of the mother during the entire pregnancy. Finally, the intensity of alcohol use before pregnancy appears to be a protective indicator; that is, women who smoked during the entire pregnancy were more likely to have a lower intensity of alcohol use before pregnancy than women who did not smoke during the entire pregnancy.

\section{Indicators Associated with Women who Successfully}

Quit Smoking during Pregnancy

Table 3 presents the BM and MM of risk and protective indicators associated with women who successfully quit smoking during pregnancy. The BM showed that women who did (vs. did not) successfully quit smoking during pregnancy were more likely to have a higher educational level, a higher intensity of alcohol use before pregnancy, and a partner who did not smoke before and during pregnancy or who cut down on smoking during pregnancy. In addition, women who did (vs. did not) successfully quit smoking during pregnancy were less likely to have talked about smoking cessation with a health care professional or used smoking cessation support during pregnancy. In the MM, a high educational level and a higher intensity of alcohol use before pregnancy remained significantly associated with successfully quitting during pregnancy, as well as a lower rate of talking with a health care professional and no use of smoking cessation support.

\section{Indicators Associated with Women who \\ Relapsed Postpartum}

According to the $\mathrm{BM}$, maternal age was significantly related to relapsing postpartum. In addition, women who relapsed postpartum more often had a partner who continued smoking postpartum or who did not smoke at all (versus a partner who quit smoking postpartum) compared to women who did not relapse postpartum (Table 3). In the $\mathrm{MM}$, only the partner's smoking behavior postpartum remained significant. It appears that women who relapsed postpartum more often had a partner who continued smoking postpartum or a partner who did not smoke at all compared to women who did not relapse postpartum.

\section{Discussion/Conclusion}

This study offers a different and unique perspective by drawing together risk and protective indicators for before, during, and after pregnancy as a combined analysis in one single sample. Moreover, a broad set of indicators was ex- 
Table 2. Indicators associated with women who smoked before pregnancy $(n=311)$ versus women who did not smoke before pregnancy $(n=1,547)$ and with women who smoked during the entire pregnancy $(n=84)$ versus women who did not smoke during the entire pregnancy $(n=1,765)$

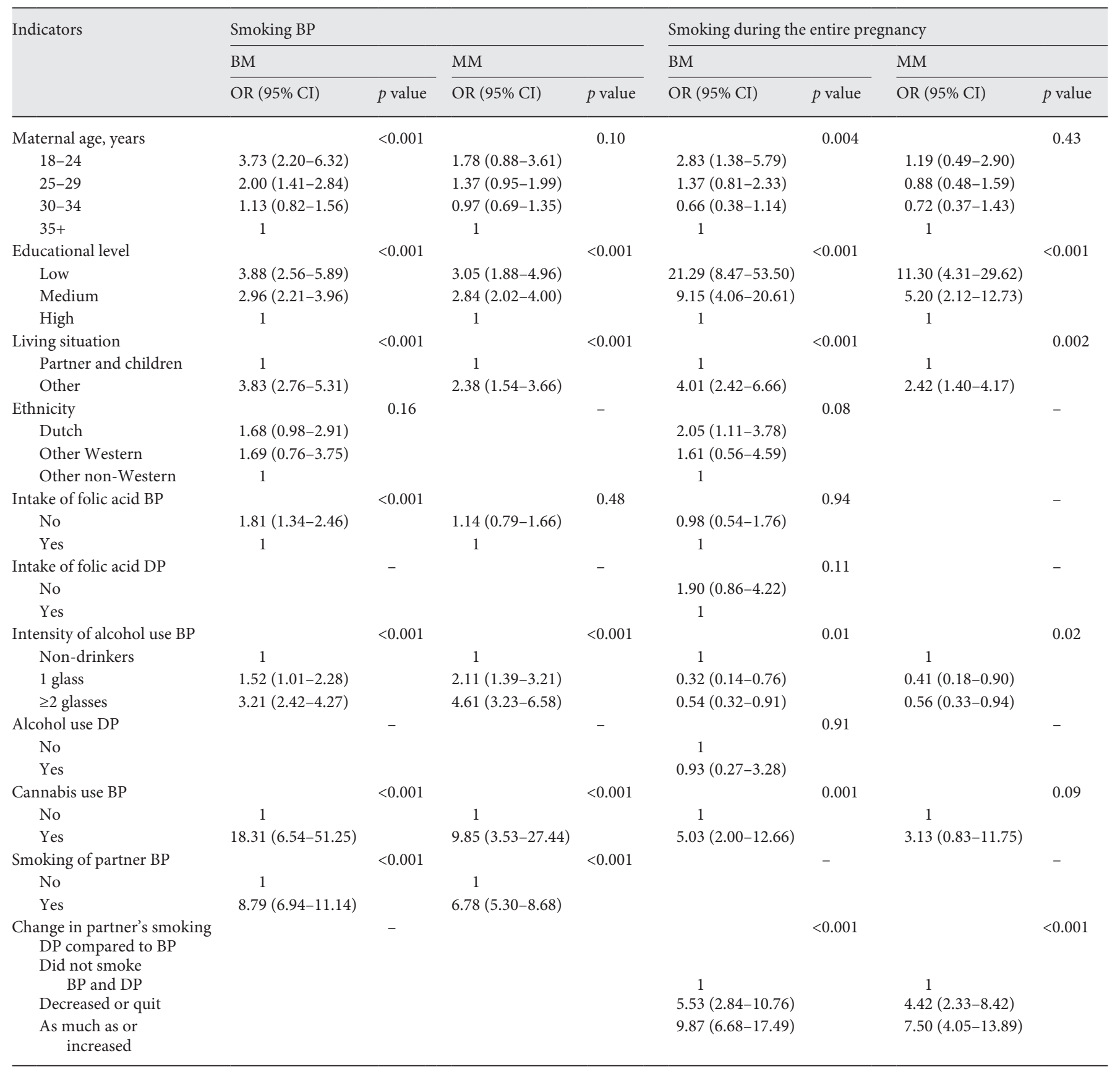

The outcome variables were categorized as "smoking four weeks before pregnancy" (yes = 1; no =0) and "smoking during the entire pregnancy (i.e., during all three trimesters)" (yes $=1$; no $=0$ ).

$\mathrm{BM}$, bivariate model; MM, multivariate model; BP, before pregnancy; $\mathrm{DP}$, during pregnancy; $\mathrm{PP}$, postpartum. 


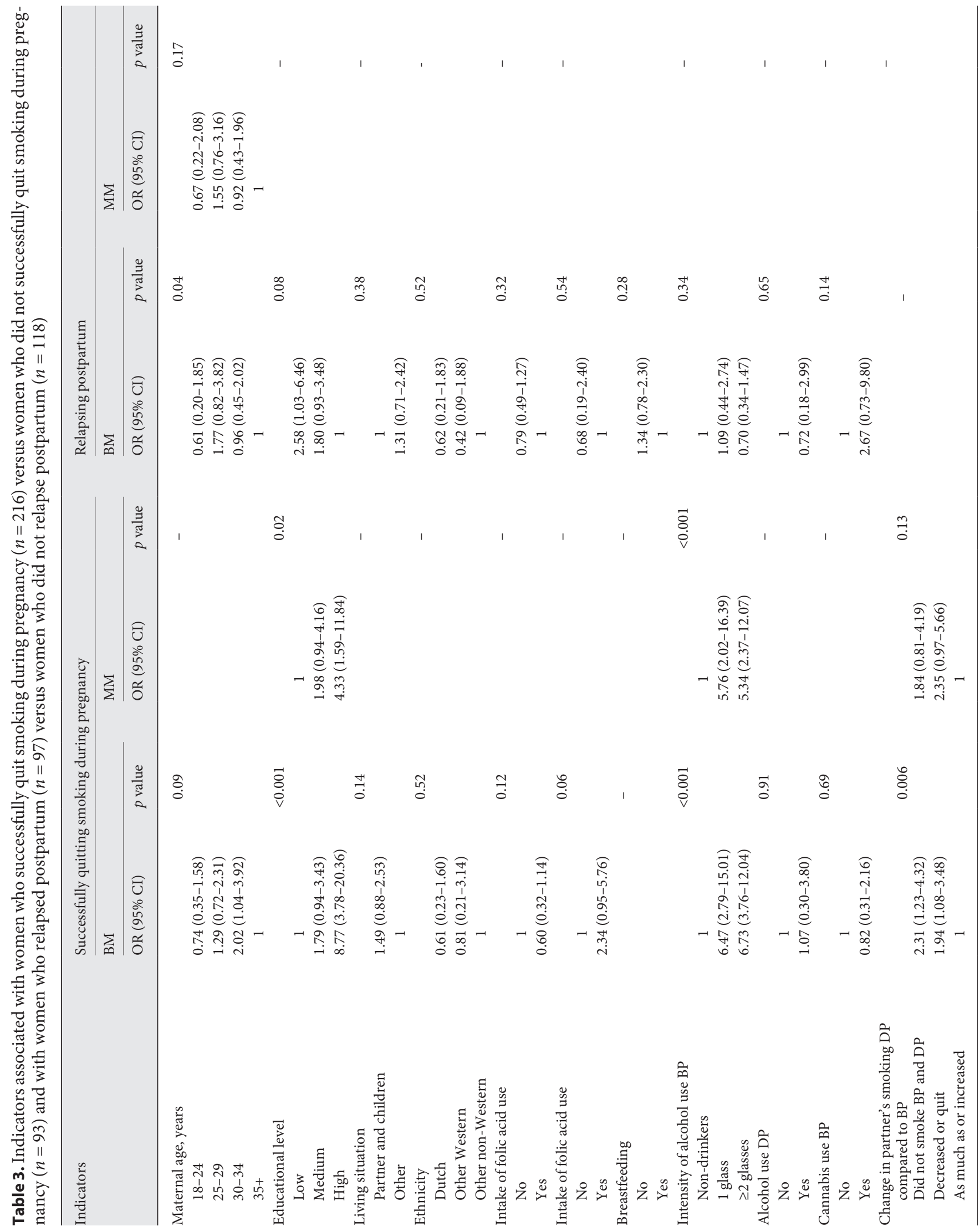




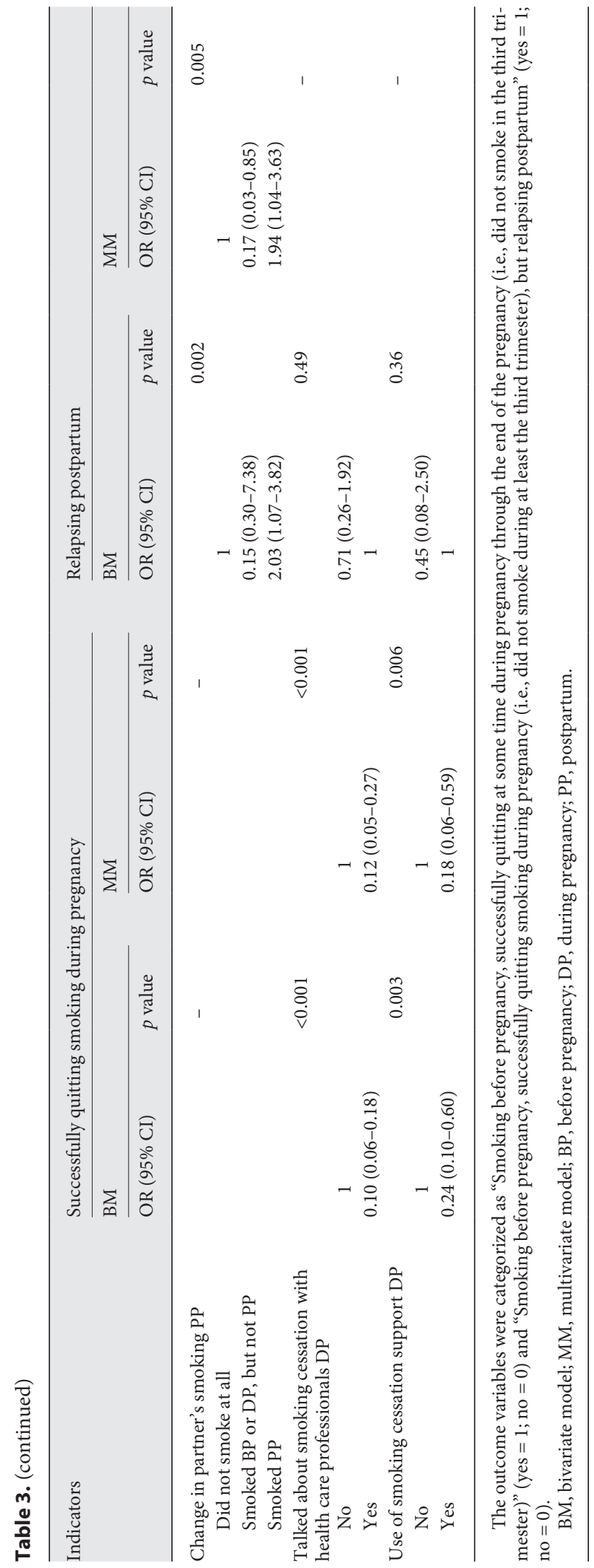

amined, including sociodemographic indicators, women's substance use, smoking behavior of the partner around pregnancy, and use of smoking cessation support. A major finding of this study is that the relationship between the partner's smoking status and the smoking status of women around pregnancy cannot be underestimated. Women who smoked before pregnancy were nearly 7 times more likely to have a partner who smoked before pregnancy compared to women who did not smoke before pregnancy. Similarly, two thirds of the women who smoked during the entire pregnancy also had a partner who smoked during pregnancy. Women who did (vs. did not) smoke during the entire pregnancy were 7.5 times more likely to have a partner who smoked as much as before pregnancy or increased smoking during pregnancy. In addition, women who did (vs. did not) smoke during the entire pregnancy were nearly 4.5 times more likely to have a partner who decreased their smoking or quit smoking during pregnancy. Finally, women who did (vs. did not) relapse postpartum were nearly twice as likely to have a partner who continued smoking postpartum. These findings are consistent with prior studies $[24,25,27,32,37]$ and provide more insight into the relationship between the timing of the partner's smoking behavior and maternal smoking status around pregnancy (e.g., women who smoked during the entire pregnancy were nearly 4.5 times more likely to have a partner who decreased their smoking or quit smoking during pregnancy compared to women who did not smoke during the entire pregnancy).

Another important finding of this study is the one that highlights the profound association between women's educational level and their smoking status before and during pregnancy. This study found that women who did (vs. did not) smoke before pregnancy were about 3 times more likely to have a low or medium educational level. In addition, women who did (vs. did not) smoke during the entire pregnancy were nearly 11.5 times more likely to have a low educational level and more than 5 times more likely to have a medium educational level. Conversely, women who did (vs. did not) successfully quit smoking during pregnancy were more than 4 times more likely to be highly educated. These findings are in line with previous studies [21, 23, 25-27, 34] and emphasize that more attention is needed to help women with a lower educational level to quit smoking before and during pregnancy.

With respect to the impact of maternal substance use on smoking around pregnancy, our findings showed that women who did (vs. did not) smoke before pregnancy were nearly 10 times more likely to have also used cannabis before pregnancy. This study adds to the evidence that cannabis and tobacco are concurrently used among 
women around pregnancy $[38,39]$. The role of intensity of alcohol use before pregnancy on smoking around pregnancy was ambiguous. On the one hand, women who did (vs. did not) smoke before pregnancy were more likely to have a higher intensity of alcohol use before pregnancy. On the other hand, women who did (vs. did not) successfully quit smoking during pregnancy were more likely to have a higher intensity of alcohol use before pregnancy. Although women's educational level might play a role here (more educated individuals tend to drink more alcohol than less educated individuals) [40], the MM demonstrated that the relationship between intensity of alcohol use and women's smoking status is maintained even when taking women's educational level into account. Therefore, it is difficult to comprehend these mixed results, and more research is needed on the relationship between women's intensity of alcohol use before pregnancy and women's smoking behavior around pregnancy.

Two other contrasting findings of this study showed that women who did (vs. did not) successfully quit smoking during pregnancy had talked about smoking cessation with a health care professional during pregnancy less often and also used smoking cessation support during pregnancy less often. However, a Cochrane review revealed that smokers who quit smoking more often talked about smoking cessation with health care professionals [41]. In addition, research has shown that smokers are much more likely to quit smoking when they receive smoking cessation support (e.g., telephone counseling) [42]. Our findings could be caused by a selection effect. That is, it may be the case that health care professionals mostly talked about smoking cessation with women who were more addicted to smoking and therefore found it more difficult to quit smoking. Likewise, smoking cessation support may have been mostly used by women who were more addicted to smoking.

\section{Strengths and Limitations}

A major strength of this study concerns the recruitment strategy. In the Netherlands, nearly all parents visit YHC centers regularly so that their children's physical and social development can be monitored [36]. Because women were recruited at YHC centers that were randomly selected at the city level, this study was able to recruit a sample that was largely representative of Dutch women with young children. Another strength is that we included some indicators (e.g., change in partner's smoking during and after pregnancy) in the analyses that, as far as we know, have not been examined before. Because of this, we were able to examine whether any change in partners'

Indicators of Women's Smoking Behavior before, During, and after Pregnancy smoking around pregnancy affected women's smoking status around pregnancy. This investigation resulted in some new insights concerning the association between partners' smoking behavior and women's smoking status around pregnancy (e.g., women were less likely to smoke during the entire pregnancy when their partner decreased their smoking or quit smoking during pregnancy compared to women whose partner smoked as much as or increased their smoking during pregnancy). These insights could be important for the development and/or improvement of smoking cessation and relapse prevention interventions that are tailored to this specific group. A limitation of this study is that the data were collected at one moment (i.e., cross-sectional), so no causal relations can be concluded. In addition, two of the examined indicators (i.e., alcohol use during pregnancy and change in partner's smoking postpartum) had cell values $<5$ in some of the investigated groups (e.g., women who relapsed postpartum). Therefore, caution is needed in interpreting these results. A third limitation is that the data were selfreported and not biochemically validated. Biochemical validation was impossible due to the study design. In selfreport, an important source of underreporting is related to the social-desirability bias [43], which should be minimized as far as possible. The fact that $94.2 \%$ of the women who were approached agreed to participate in this study illustrates that they were willing to provide answers on this sensitive topic. Moreover, the questionnaires were completely anonymous (i.e., names and contact information were not collected) and the women filled in the questionnaires by themselves to further decrease such bias. A fourth limitation is that the data were retrospectively obtained, which could make them subject to recall bias. However, several studies have provided evidence that recall on smoking behavior during pregnancy is quite accurate $[44,45]$. Ergin et al. [44] assumed that this accuracy is a result of the social stigma associated with smoking during pregnancy and the fact that pregnancy is experienced as an important life event.

\section{Implications for Practice}

This study emphasizes the importance of having a smoke-free partner at all points around pregnancy. More specifically, our results suggest that it is essential that partners who smoke quit smoking before pregnancy and do not smoke during pregnancy. Still, when partners are not able or willing to quit smoking before or during pregnan$c y$, it is pivotal for partners to be encouraged to quit smoking postpartum, since women who relapsed postpartum more often had a partner who continued smoking post- 
partum compared to women who did not relapse postpartum. Based on these findings, it might not be sufficient for health care professionals to address partners' smoking and provide them with effective cessation support exclusively during pregnancy. Instead, it is necessary for health care professionals to also address partners' smoking behavior and provide them with evidence-based cessation support before and after pregnancy. A systematic review found 9 studies that tested smoking cessation interventions for partners of pregnant women [46]. Only two of these studies (in which partners received, e.g., nicotine replacement therapy) yielded positive results on partner's smoking cessation $[47,48]$. However, there was no evidence that these results were maintained postpartum (i.e., one study did not examine this [47], and the other study did not report these results [48]). The postpartum period could serve as a key time-point to provide partners smoking with cessation interventions, since at this time-point partners have a heightened awareness that being a smoker is in conflict with being a "good parent"[49]. Thus, further research should not exclusively focus on developing and examining smoking cessation interventions for partners during pregnancy. Instead, the main focus of further intervention research should be to examine how partners can abstain from smoking before and after pregnancy.

Finally, this study underlines that women who smoked before pregnancy more often used cannabis and/or had a high intensity of alcohol use before pregnancy than women who did not smoke before pregnancy. Research showed that concurrently continuing to both smoke and use other substances (such as alcohol and cannabis) during pregnancy can have serious adverse health effects for women's pregnancies and their fetuses [50]. In many cases, women do not know that they are pregnant during the first few weeks of their pregnancy. Therefore, in order to limit women's concurrent substance use during these early weeks of pregnancy, it is important that women of childbearing age who use substances concurrently receive information on the importance of quitting smoking and other substances before pregnancy.

\section{Acknowledgement}

Not applicable.

\section{Ethics Statement}

Participants have given their written informed consent. In addition, the Ethics Committee of the Trimbos Institute has approved the study protocol of the Dutch Monitor on Substance Use and Pregnancy.

\section{Disclosure Statement}

The authors declare that they have no conflicts of interest to disclose.

\section{Funding Sources}

This work was supported by the Dutch Ministry of Health, Welfare, and Sport and the Dutch Cancer Society (grant number: 2015-7944). The funding bodies had no role in the design of the study and collection, analysis, and interpretation of data, and in writing the manuscript.

\section{Authors Contribution}

M.T. was responsible for the recruitment and data collection. T.S.S. was responsible for the analyses and report of the study results. R.O., R.E., and M.K. were supervisors. All authors read and approved of the final manuscript.

\section{References}

1 Riaz M, Lewis S, Naughton F, Ussher M. Predictors of smoking cessation during pregnancy: a systematic review and meta-analysis. Addiction. 2018 Apr;113(4):610-22.

2 Levitt C, Shaw E, Wong S, Kaczorowski J; McMaster University Postpartum Research Group. Systematic review of the literature on postpartum care: effectiveness of interventions for smoking relapse prevention, cessation, and reduction in postpartum women. Birth. 2007 Dec;34(4):341-7.

3 Hofhuis W, de Jongste JC, Merkus PJ. Adverse health effects of prenatal and postnatal tobacco smoke exposure on children. Arch Dis Child. 2003 Dec;88(12):1086-90.

4 Ekblad M, Korkeila J, Lehtonen L. Smoking during pregnancy affects foetal brain development. Acta Paediatr. 2015 Jan;104(1):128.

5 Ekblad M, Gissler M, Lehtonen L, Korkeila J. Prenatal smoking exposure and the risk of psychiatric morbidity into young adulthood. Arch Gen Psychiatry. 2010 Aug;67(8):8419.

6 Williams GM, O'Callaghan M, Najman JM, Bor W, Andersen MJ, Richards D, et al. Ma- ternal cigarette smoking and child psychiatric morbidity: a longitudinal study. Pediatrics. 1998 Jul;102(1):e11.

7 DiFranza JR, Aligne CA, Weitzman M. Prenatal and postnatal environmental tobacco smoke exposure and children's health. Pediatrics. 2004 Apr;113(4 Suppl):1007-15.

8 Leonardi-Bee J, Jere ML, Britton J. Exposure to parental and sibling smoking and the risk of smoking uptake in childhood and adolescence: a systematic review and meta-analysis. Thorax. 2011 Oct;66(10): 847-55. 
9 McBride CM, Emmons KM, Lipkus IM. Understanding the potential of teachable moments: the case of smoking cessation. Health Educ Res. 2003 Apr;18(2):156-70.

10 Crozier SR, Robinson SM, Borland SE, Godfrey KM, Cooper C, Inskip HM; SWS Study Group. Do women change their health behaviours in pregnancy? Findings from the Southampton Women's Survey. Paediatr Perinat Epidemiol. 2009 Sep;23(5):44653.

11 Caleyachetty R, Tait CA, Kengne AP, Corvalan C, Uauy R, Echouffo-Tcheugui JB. Tobacco use in pregnant women: analysis of data from Demographic and Health Surveys from 54 low-income and middle-income countries. Lancet Glob Health. 2014 Sep; 2(9):e513-20.

12 Jones M, Lewis S, Parrott S, Wormall S, Coleman T. Re-starting smoking in the postpartum period after receiving a smoking cessation intervention: a systematic review. Addiction. 2016 Jun;111(6):981-90.

13 Donatelle RJ, Prows SL, Champeau D, Hudson D. Randomised controlled trial using social support and financial incentives for high risk pregnant smokers: significant other supporter (SOS) program. Tob Control. 2000; 9(90003 Suppl 3):III67-9.

14 Hannöver W, Thyrian JR, Röske K, Grempler J, Rumpf HJ, John U, et al. Smoking cessation and relapse prevention for postpartum women: results from a randomized controlled trial at $6,12,18$ and 24 months. Addict Behav. 2009 Jan;34(1):1-8.

15 Reitzel LR, Vidrine JI, Businelle MS, Kendzor DE, Costello TJ, Li Y, et al. Preventing postpartum smoking relapse among diverse low-income women: a randomized clinical trial. Nicotine Tob Res. 2010 Apr;12(4):32635.

16 Chamberlain C, O’Mara-Eves A, Porter J, Coleman T, Perlen SM, Thomas J, et al. Psychosocial interventions for supporting women to stop smoking in pregnancy. Cochrane Database Syst Rev. 2017 Feb;2: CD001055

17 Hajek P, Stead LF, West R, Jarvis M, Hartmann-Boyce J, Lancaster T. Relapse prevention interventions for smoking cessation. Cochrane Database Syst Rev. 2013 Aug;(8) CD003999.

18 Krebs P, Prochaska JO, Rossi JS. A meta-analysis of computer-tailored interventions for health behavior change. Prev Med. 2010 SepOct;51(3-4):214-21.

19 Noar SM, Benac CN, Harris MS. Does tailoring matter? Meta-analytic review of tailored print health behavior change interventions. Psychol Bull. 2007 Jul;133(4):673-93.

20 Lustria ML, Noar SM, Cortese J, Van Stee SK, Glueckauf RL, Lee J. A meta-analysis of webdelivered tailored health behavior change interventions. J Health Commun. 2013;18(9): 1039-69.

21 Smedberg J, Lupattelli A, Mårdby AC, Nordeng H. Characteristics of women who continue smoking during pregnancy: a cross-sectional study of pregnant women and new mothers in 15 European countries. BMC Pregnancy Childbirth. 2014 Jun;14(1): 213.

22 Mohsin M, Bauman AE. Socio-demographic factors associated with smoking and smoking cessation among 426,344 pregnant women in New South Wales, Australia. BMC Public Health. 2005 Dec;5(1):138.

23 Nur N. Association of risk factors with smoking during pregnancy among women of childbearing age: an epidemiological field study in Turkey. Sao Paulo Med J. 2017 Mar-Apr; 135(2):100-6.

$24 \mathrm{Xu} \mathrm{H}$, Wen LM, Rissel C, Baur LA. Smoking status and factors associated with smoking of first-time mothers during pregnancy and postpartum: findings from the Healthy Beginnings Trial. Matern Child Health J. 2013 Aug;17(6):1151-7.

25 Penn G, Owen L. Factors associated with continued smoking during pregnancy: analysis of socio-demographic, pregnancy and smokingrelated factors. Drug Alcohol Rev. 2002 Mar; 21(1):17-25.

26 Härkönen J, Lindberg M, Karlsson L, Karlsson H, Scheinin NM. Education is the strongest socio-economic predictor of smoking in pregnancy. Addiction. 2018 Jun;113(6): 1117-26.

27 Kia F, Tosun N, Carlson S, Allen S. Examining characteristics associated with quitting smoking during pregnancy and relapse postpartum. Addict Behav. 2018 Mar;78:114119.

28 Connor SK, McIntyre L. The sociodemographic predictors of smoking cessation among pregnant women in Canada. Can J Public Health. 1999 Sep-Oct;90(5):352-5.

$29 \mathrm{Lu} \mathrm{Y}$, Tong S, Oldenburg B. Determinants of smoking and cessation during and after pregnancy. Health Promot Int. 2001 Dec;16(4): 355-65.

30 Polanska K, Hanke W, Sobala W, Lowe JB, Jaakkola JJ. Predictors of smoking relapse after delivery: prospective study in central Poland. Matern Child Health J. 2011 Jul;15(5): 579-86.

31 Solomon LJ, Higgins ST, Heil SH, Badger GJ, Thomas CS, Bernstein IM. Predictors of postpartum relapse to smoking. Drug Alcohol Depend. 2007 Oct; $90(2-3): 224-7$.

32 Simmons VN, Sutton SK, Quinn GP, Meade $\mathrm{CD}$, Brandon TH. Prepartum and postpartum predictors of smoking. Nicotine Tob Res. 2014 Apr;16(4):461-8.

33 Colman GJ, Joyce T. Trends in smoking before, during, and after pregnancy in ten states. Am J Prev Med. 2003 Jan;24(1):29-35.

34 Kahn RS, Certain L, Whitaker RC. A reexamination of smoking before, during, and after pregnancy. Am J Public Health. 2002 Nov; 92(11):1801-8.

35 Tuithof M, Siauw R, Van Dorsselaer S, Monshouwer K. Factsheet Monitor Zwangerschap en Middelengebruik Het middelengebruik van moeders en hun partner voor, tijdens en na de zwangerschap. Utrecht; 2017. Available from: https://assets.trimbos.nl/docs/3600fc07b31b-4505-9664-6d48ec07eadc.pdf.

36 Nederlands Centrum Jeugdgezondheid (NCJ). Landelijk professioneel kader: Uitvoering basispakket jeugdgezondheidszorg (JGZ). Utrecht; 2018. Available from: https:// assets.ncj.nl/docs/9c8aba38-2e8d-4fef-a346ab7dab7f8bc3.pdf.

37 Orton S, Coleman T, Coleman-Haynes T, Ussher M. Predictors of Postpartum Return to Smoking: A Systematic Review. Nicotine Tob Res. 2018 May;20(6):665-73.

38 Passey ME, Sanson-Fisher RW, D'Este CA, Stirling JM. Tobacco, alcohol and cannabis use during pregnancy: clustering of risks. Drug Alcohol Depend. 2014 Jan; 134 44-50.

39 El Marroun H, Tiemeier H, Jaddoe VW, Hof man A, Mackenbach JP, Steegers EA, et al. Demographic, emotional and social determinants of cannabis use in early pregnancy: the Generation R study. Drug Alcohol Depend. 2008 Dec;98(3):218-26.

40 van Laar MW, van Gestel B, Cruts AA, van der Pol PM, Ketelaars AP, Beenakkers EM, et al. Nationale Drug Monitor: Jaarbericht 2017. Utrecht; 2017. Available from: www. wodc.nl.

41 Stead LF, Buitrago D, Preciado N, Sanchez G, Hartmann-Boyce J, Lancaster T. Physician advice for smoking cessation. Cochrane Database Syst Rev. 2013 May;(5): CD000165

42 Stead LF, Koilpillai P, Fanshawe TR, Lancaster T. Combined pharmacotherapy and behavioural interventions for smoking cessation. Cochrane Database Syst Rev. 2016 Mar 24 3:CD008286.

43 Schneider S, Schütz J. Who smokes during pregnancy? A systematic literature review of population-based surveys conducted in developed countries between 1997 and 2006 Eur J Contracept Reprod Health Care. 2008 Jun;13(2):138-47.

44 Ergin I, Hassoy H, Tanik FA, Aslan G. Maternal age, education level and migration: socioeconomic determinants for smoking during pregnancy in a field study from Turkey. BMC Public Health. 2010 Jun;10(1): 325

45 Hensley Alford SM, Lappin RE, Peterson L, Johnson CC. Pregnancy associated smoking behavior and six year postpartum recall. Matern Child Health J. 2009 Nov; 13(6): $865-72$.

46 Hemsing N, Greaves L, O’Leary R, Chan K, Okoli C. Partner support for smoking cessation during pregnancy: a systematic review. Nicotine Tob Res. 2012 Jul;14(7):76776.

47 Stanton WR, Lowe JB, Moffatt J, Del Mar CB. Randomised control trial of a smoking cessation intervention directed at men whose partners are pregnant. Prev Med. 2004 Jan;38(1): 6-9. 
48 McBride CM, Baucom DH, Peterson BL, Pollak KI, Palmer C, Westman E, et al. Prenatal and postpartum smoking abstinence a partner-assisted approach. Am J Prev Med. 2004 Oct;27(3):232-8.
49 Flemming K, Graham H, McCaughan D, Angus K, Bauld L. The barriers and facilitators to smoking cessation experienced by women's partners during pregnancy and the post-partum period: a systematic review of qualitative research. BMC Public Health. 2015 Sep;15(1): 849.
50 Viteri OA, Soto EE, Bahado-Singh RO, Christensen CW, Chauhan SP, Sibai BM. Fetal anomalies and long-term effects associated with substance abuse in pregnancy: a literature review. Am J Perinatol. 2015 Apr;32(5):40516. 